\title{
A solar station in Ica - Mutsumi Ishitsuka: a research center to improve education at the university and schools
}

\author{
Raúl Terrazas-Ramos \\ Facultad de Ciencias, Universidad Nacional San Luis Gonzaga de Ica, Perú \\ email: raulterrazas81@gmail.com
}

\begin{abstract}
The San Luis Gonzaga National University of Ica has built a solar station, in collaboration with the Geophysical Institute of Peru, the National Astronomical Observatory of Japan and the Hida Observatory. The Solar Station has the following equipment: a digital Spectrograph Solar Refractor Telescope Takahashi $15 \mathrm{~cm}$ aperture, $60 \mathrm{~cm}$ reflector telescope aperture, a magnetometer-MAGDAS / CPNM and a Burst Monitor Telescope Solar-FMT (Project CHAIN ). These teams support the development of astronomical science and Ica in Peru, likewise contributing to science worldwide. The development of basic science will be guaranteed when university students, professors and researchers work together. The Solar Station will be useful for studying the different levels of university education and also for the general public. The Solar Station will be a good way to spread science in the region through public disclosure.
\end{abstract}

Keywords. instrumentation: miscellaneous, methods: data analysis

\section{Introduction}

In 1998, the Faculty of Science at the University of San Luis Gonzaga Ica (UNICA) sought the help of physics lab equipment, the Ancon Observatory Geophysical Institute of Peru (IGP, Itshisuka 2006, Ishitsuka et al. 2007). From this date a joint activity was begun between the UNICA and the IGP. In 2002 a Takahashi telescope with electronic drive and an equatorial mount $150 \mathrm{~mm}$ aperture and focal length of $1050 \mathrm{~mm}$ was installed on the roof of the Faculty of Sciences. Images from the Sun by the projection method have been obtained. A group of students from the Faculty of Sciences was trained to obtain the relative sunspot number, and the first results of the observations were published in a bulletin by the IGP in 2004. In 2003, the UNICA bought a digital camera, Nikon Coolpix 5000 series in order to photograph the solar photosphere. In 2004 the IGP signed a cooperation agreement with the National Astronomical Observatory of Japan (NAOJ), achieving mutual cooperation in the fields of solar physics, astronomy and radio astronomy. Then at the end of 2005, the UNICA decided to give 4.2 acres within the campus of the University for the construction of the Solar Station. In March 2010 Ica Solar Station hosted the following teams: a solar spectrograph, a magnetometer, a 60 $\mathrm{cm}$ reflecting telescope and the FMT whose main objective is to establish a network of telescopes, with similar characteristics, installed around the world to monitor the Sun (Project CHAIN-International Network for Continuous Observation of the Sun in $\mathrm{H}$-alpha Images).

\section{Solar station}

Owing to the good quality of the sky, and the number of hours of sunshine that characterize the city of Ica, it was decided to build the Solar Station. The Solar Station is 
located within the campus in the city of Ica. This city is located south of Lima. This station is the first observatory of its kind in Perú. The Solar Station construction was funded by UNICA. The Geophysical Institute of Perú, the National Astronomical Observatory of Japan and the Hida Observatory of Kyoto University provide the equipment to be installed at the Solar Station.

Location:

Latitude: $140^{\circ} 05^{\prime} 20.55^{\prime \prime} \mathrm{S}$

Longitude: $75^{\circ} 44^{\prime} 11.28^{\prime \prime} \mathrm{W}$

Altitude: $406 \mathrm{~m}$

$300 \mathrm{~km}$ south of Lima

Weather: Annual precipitation: $3 \mathrm{~mm}$ in average

Average temperature: $23{ }^{\circ} \mathrm{C}$

Average humidity: $20 \%$

Never snows

Equipment:

Digital spectroheliograph

FMT, Flare Monitor Telescope

Reflector Telescope $60 \mathrm{~cm}$

Refractor Telescope Takahashi $15 \mathrm{~cm}$ (located on the roof of the Faculty of Science at the University of San Luis Gonzaga Ica)

Magnetometer MAGDAS

\section{Research at the Solar Station}

Digital spectroheliograph: this instrument can measure the properties of the emission lines and several absorption lines that occur in the solar atmosphere and thus is able to determine the equation of state characterizing the plasma which is part of the chromosphere and solar photosphere. It uses a coelostat to collect sunlight throughout the day.

Study of solar activity through the data captured and analyzed using mathematical models of the relative sunspot number. Currently records of sunspots by the projection method are being made with the Takahashi refractor telescope of $15 \mathrm{~cm}$ of aperture on the roof of the Faculty of Sciences. These records are made each day and are sent to the Ancon Observatory where they are processed to determine the Wolf number for each of the observers, and verify the reliability of the sunspot records. The Wolf number (R) of each observer's data are compared with those obtained in the Solar Influence Data Analysis Center (SIDC), Brussels. It also is conducting analysis and creating a database of sunspots, which will allow us to find a systematic methodology for the evolution of sunspot active regions, and also look for a technique to evaluate the consistency of the data quality of sunspots and calculate the area of active regions with sunspots.

FMT - Telescope Flares monitored (solar flares): The FMT is a telescope to observe the Sun in H-alpha and it is part of the international project CHAIN (Continuous Halpha Imaging Network, Ueno 2007) to continuously monitor the Sun in Earth-based observatories. The FMT consists of 5 solar telescopes that simultaneously observe the entire solar disk at different wavelengths around the H-alpha line. Furthermore, the FMT can measure the velocity field in three-dimensional structures that move over the entire solar disk. The scientific objective of the FMT is to monitor solar flares and 
eruptive filaments continuously over the whole solar disk and investigate the correlation between the characteristics of these eruptive phenomena and the corresponding efficiency of Coronal Mass Ejections (CMEs).

\section{Conclusions}

It is important to carry out this joint project between UNICA, Hida Observatory, NAOJ and the IGP, and contribute to the development of solar physics in Ica and Perú.

The development of basic science will be guaranteed when university students, professors and researchers work together.

The Solar Station of Ica is very useful for the study at different levels of university education and also of interest to the general public.

\section{Acknowledgements}

Special thanks to Dr. Mutsumi Ishitsuka who was Director of the Ancon-IGP, who initiated this unique and important project to develop the Sslar physics in our region. Thanks to the authorities of the Universidad Nacional San Luis Gonzaga de Ica for promoting the development of the Solar Station of Ica.

\section{References}

Ishitsuka, J. K. et al. 2007, Bulletin of the Astronomical Society of India, 35. 709

Ishitsuka, J. K. 2006, Proceedings of the $2^{\text {nd }} U N / N A S A$ Workshop on International Heliophysical Year and Basic Space Science, 76

Ueno, S. 2007, Bulletin of the Astronomical Society of India, 35, 6 